\title{
Flexible Robot-Assembly using a Multi-Sensory Approach
}

\author{
Stefan Jörg, Jörg Langwald, \\ Johannes Stelter, Gerd Hirzinger \\ German Aerospace Center \\ Institute of Robotics and System Dynamics \\ 82234 Wessling, Germany \\ Johannes.Stelter@dlr.de
}

\begin{abstract}
Recent research in industrial robotics aims at the involvement of additional sensoric devices to improve robustness, flexibility and performance of common robot applications. Many different sensors have been developed over the past years to fit the requirements of different but very specific tasks. Special seam tracking sensors support the robot in welding applications. Vision systems are common in quality control and inspection. Force/torque sensors mounted to a robot's wrist are still an exception and limited to the fields of scientific research. Compared to the number of annual robot sales the number of sensor equipped robots is still negligible, although the benefits of sensoric feedback are obvious. In this paper we introduce a general approach to tackle the problem of sensor-based robot assembly. We realized a flexible assembly cell that includes a variety of different sensors for mating with moving parts.
\end{abstract}

\section{Introduction}

Higher requirements in all areas of industrial production directly initiate the development of more sophisticated automation systems. Due to the time-is-money rules of industrial productions huge efforts are made by the robot vendors to improve the speed and precision of their systems. But beside the kinematic capabilities of the robot the sensing abilities are still very underdeveloped. Nevertheless good sensing abilities will be absolutely necessary to gain a higher flexibility and autonomy of robots. These features will be a crucial factor to meet the upcoming requirements in production:

\author{
Ciro Natale
}

PRISMA Lab

Dipartimento di Informatica e Sistemistica Università degli Studi di Napoli Federico II

Via Claudio 21, 80125 Napoli, Italy

cinatale@unina.it

- uncertainties in positions of tool and/or workpiece

- accessory parts that guarantee a fixed position of the workpiece like feeders and fixtures are expensive and mostly adjusted to one single workpiece. Therefore these devices are to be substituted by more flexible solutions

- tool attrition results in a slight movement of the tool coordinate system which has to be compensated by the robot

- due to new production strategies robots have to operate on moving workpieces

- evaluation of interacting forces and torques

- protects tool, workpiece and the robot itself from damage

- force/torque feedback (sense of touch) is the basis for designing solution strategies for high-level robot applications.

To demonstrate the effectiveness and robustness of our methods we decided to set up a flexible automation cell which includes all features and technologies that have been developed over the past years at our institute. Beside the scientific challenge we explicitly aspired to robustness and easy operation.

\section{The Flexible Assembly Cell}

The cell design was determined by the following factors: 
- The cell should demonstrate the abilities of sensor driven robots

- The system should be easy to operate

To solve complex assembly applications the following sensors and devices were integrated into the workcell:

- the manipulator of the cell is a KUKA KR15/2 robot and its controller KRC1. Due to the fact that KUKA robot controllers are PC-based they present itself as a carrier platform for necessary hardware and software integrations.

- image processing system consisting of a standard mono-ocular PAL camera and a VMEbus based image acquisition and processing hardware which is connected via a real-time link to the robot controller. The camera is mounted to the robot hand.

- our six degrees of freedom compliant force/torque sensor mounted to the flange of the robot

- a pneumatic gripper mounted to the compliant $\mathrm{f} / \mathrm{t}$-sensor

- an independently controlled 1-axis turntable

- additional visualization, safety and operational devices

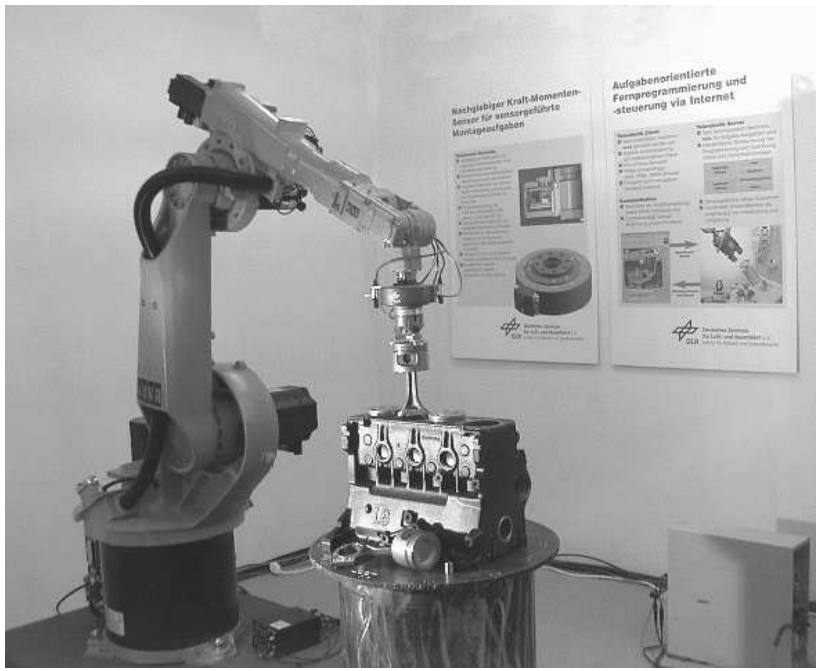

Figure 1: Overview of our assembly cell with rotating engine block and non-circular pistons

To demonstrate the abilities of our assembly cell (figure 1) we chose a setup which can roughly be described as a peg-in-moving-hole problem. A 4-cylinder diesel engine is mounted on the turntable. All four pistons together with their connecting rods are kept in a magazine. The task to be performed is defined as follows (see also figure 2):

- Take a piston from the magazine and insert it into the rotating engine block until all pistons are inserted

- remove a piston from the engine and put it back into the magazine until all pistons are stored back

The speed of the engine block is freely adjustable as long as the assembly process has not been started. There is no way to retrieve directly any information about the rotational speed of the engine and thus to set up a synchronized motion between robot and turntable. The fitting of a piston in the engine block is very tight $(<0.2 \mathrm{~mm})$ and due to the fact that pistons are slightly oval shaped to compensate thermal expansion, the insertion process is dominated by friction and jammings. Moreover the robot's wrist has to continue rotating during the whole insertion process, thus the movement constraints limit the completion time to a maximum of one and a half rotations of the engine block. The orientation of the pistons in the magazine is not fixed and therefore must be identified by the system before picking them up.

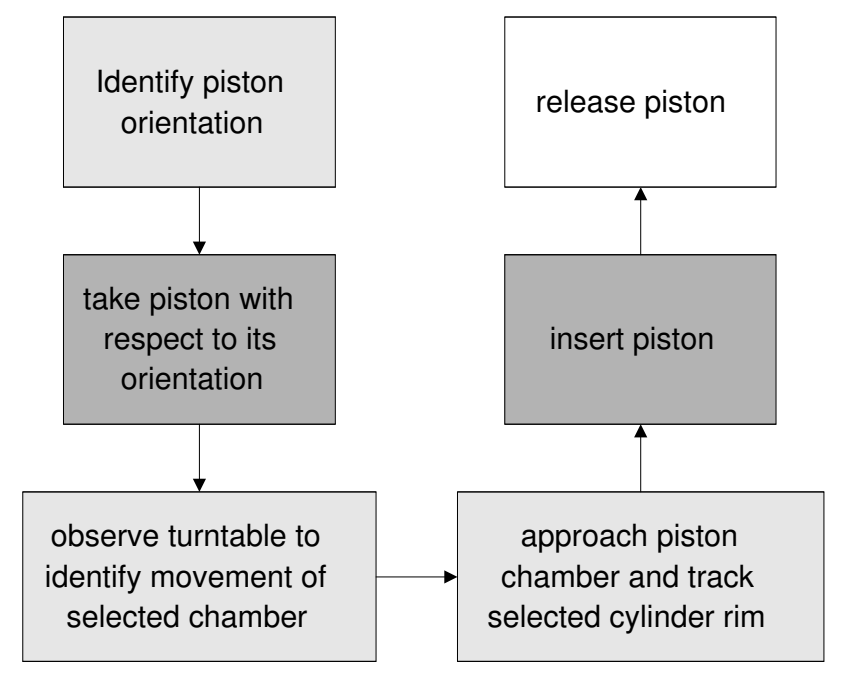

Figure 2: Assembly task order; light gray boxes illustrate vision based tasks, dark gray boxes illustrate $\mathrm{f} / \mathrm{t}$-controlled tasks 


\section{The Image Processing System}

The three main tasks for the image processing system are as follows

- detect the orientation of the piston in the magazine $\Rightarrow$ no need to fix the piston's position

- observe and detect the position and thus the movement of the engine $\Rightarrow$ plan the approach to the cylinder

- reduce the remaining position error by visual servoing just before the piston is inserted $\Rightarrow$ guarantee a valid initial position for insertion process (position error $<1 \mathrm{~mm}$ )

The camera is mounted to the robot hand. Therefore a miniaturized version of a standard PAL camera with separated electronics was chosen. The need for a large working area and thus a large viewing area resulted in the use of a wide range lens. The inevitable distortions caused by these lenses are eliminated through rectification prior to any image processing. The rectification is done by a sophisticated calibration algorithm, as described in [10], which also identifies the position of the camera relative to the robot's tool center point.

Due to the dynamic nature of the task it is essential that the image processing system operates in realtime, i.e. at least at the $25 \mathrm{~Hz}$ frame clock rate of the PAL video system. Since the observed object moves relative to the camera and the robot itself is allowed to move during observation the time delay between the acquisition of two PAL fields combined to a PAL frame results in a spatial offset. To overcome this effect each field is processed, thus resulting in a processing rate of $50 \mathrm{~Hz}$ (i.e. $20 \mathrm{~ms}$ ) and an effective pixel resolution of 384 by 287 . Where 287 is half the line numbers of a standard PAL frame and the number 384 was chosen to yield a quadratic pixel size.

To reduce the computational cost of such a high processing rate we utilized a model-based approach for all three tasks. A priori information in the form of a simplified 3D CAD model of engine and pistons is applied in a way that a complete $3 \mathrm{D}$ pose reconstruction of the engine is feasible with only one camera and by processing only predicted regions of interest (ROI) of the image. This avoids the computational overhead of a stereo system.

\section{Feature Extraction}

The extraction of geometrical object features and the determination of its parameters from camera im- ages is the computationally most intensive step in the process. The Hough-transform (HT) [5] is a robust technique to extract a large variety of geometrical object features from gradient images. Well known is its robustness against partial occlusions, variable lighting conditions, low contrast, and a patterned background. This robust approach is necessary with respect to non-continuous lighting conditions because of object and robot movement. A drawback in real-time applications is the computational complexity of the HT. Therefore an efficient implementation of the HT is necessary.

The steps of image acquisition, rectification, horizontal and vertical gradient filtering with a $7 \times 7$ kernel and the transformation in gradient magnitude and orientation values run on a Datacube MV250, a pipeline processor specialized in image preprocessing tasks. The image processing system is hosted by a PowerPC board, which also computes the HT. This flexible hardware allows the parallelization of all image processing tasks reaching a maximum rate of 12.5 to $50 \mathrm{~Hz}$, dependent on HT complexity and region size of the different operations. Details of the HT implementation used in this system are explained in [1].

Depending on the performed task and the visible object features, straight lines or circles are searched or tracked using the HT (figure 3). To speed up tracking tasks, the feature extraction step only needs to detect a small deviation from an expected feature position within a small region of interest. Feature parameters are determined by HT in a limited parameter space.

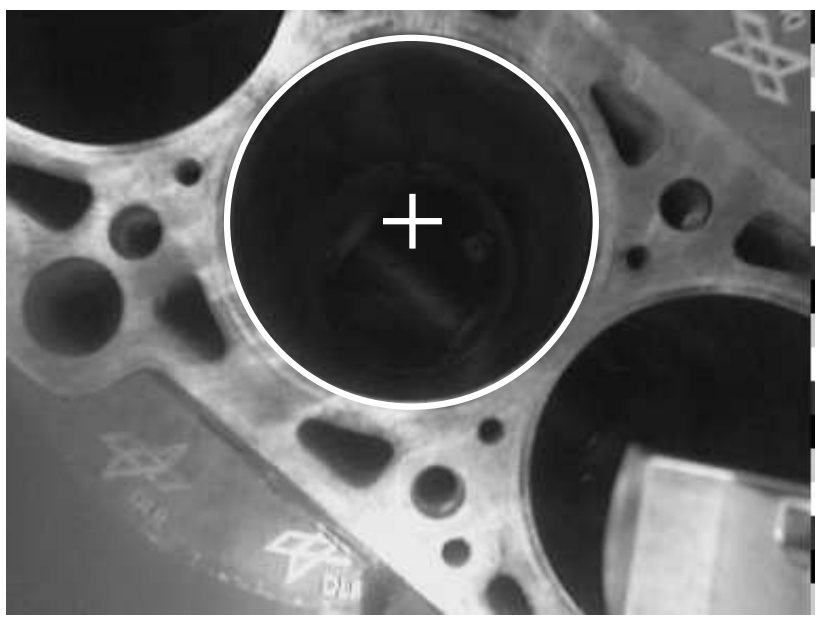

Figure 3: Tracking of the engine block approximated by a circle to reduce the remaining position error 


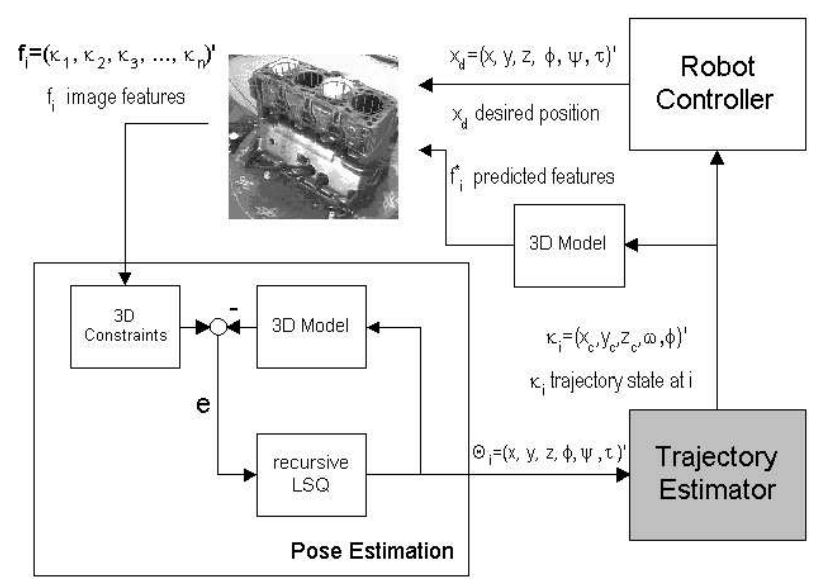

Figure 4: Tracking System Overview

\section{D Pose Estimation and Tracking}

For the computation of the position and orientation of the target from image features an extremely efficient algorithm is used.

As illustrated in figure 4 the process can be separated in two different tasks: First estimate the current $3 \mathrm{D}$ postition of the target (Pose Estimation), then predict the future position applying a known model of motion (Trajectory Estimator).

Most methods for 3D pose estimation are based on the dynamic vision approach introduced by Dickmanns [3] who suggests an extended kalman filter approach which utilizes the extracted image features as the measurement vector for the kalman filter. The drawback of this method is the dramatically increasing computational complexity for a rising number of features: $O\left(n^{3}\right)$, where $\mathrm{n}$ is the dimension of the feature vector. Since we aimed at flexibility concerning the nature of the tracked object, the number and class of the features to be tracked are not predictable. For that reason we chose a more efficient method where the complexity increases with $O(n)$, and which is up to seven times faster than other optimized algorithms[12].

Instead of minimizing a position error in the feature domain, i.e. in the $2 \mathrm{D}$ image, a pose error in $3 \mathrm{D}$ space is defined and minimized. The basic assumption is, that we experience only a small deviation from the predicted object pose, which is available by the prior output of our trajectory estimator. This results in a linear - and therefore algebraically solvable - estimation problem. For larger pose deviations - when no prediction is available - this process can be applied iteratively; in practical tracking tasks, three to four

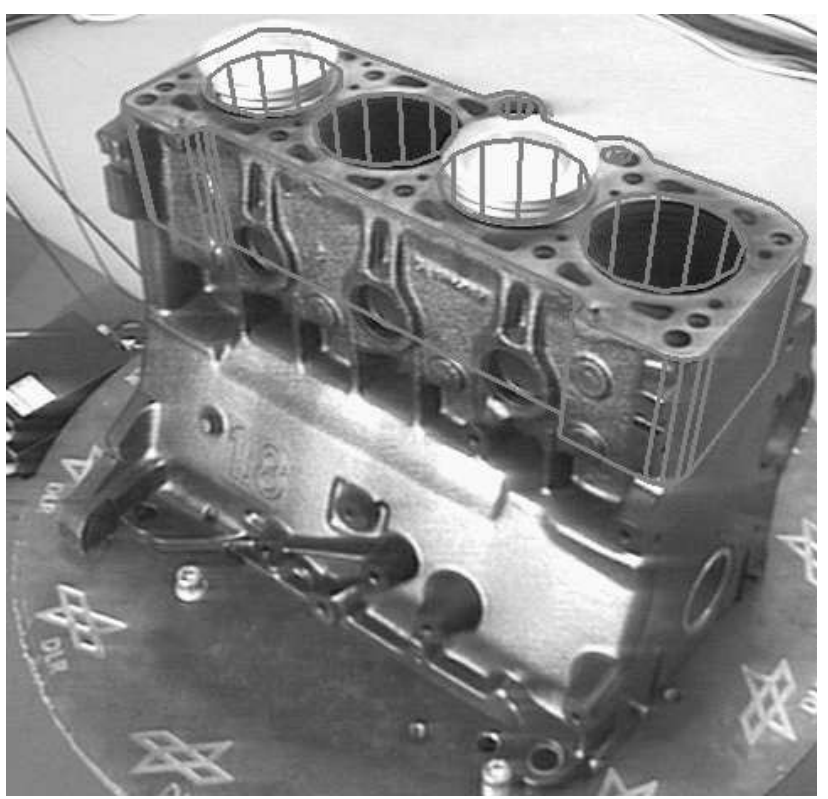

Figure 5: Visualization of the 3D pose reconstruction

iterations yield optimal alignment (see [11] for a detailed discussion). Since this method defines the pose error in the 3D domain it is possible to combine several 3D sensors, e.g. additional cameras or laser scanners. Figure 5 illustrates the visualization of the pose estimation results.

The Trajectory Estimator was implemented as a separate module. This allows the easy adaption to other tasks, i.e. to different models of target motion.

Therefore we were able to implement a very specialized Trajectory Estimator for our task: a non-linear discrete kalman filter for the estimation of a constant circular movement in a known $\mathrm{x}$-y-plane was used. It is formulated as two different states. The state in equation (1) includes the center point of the trajectory, while in (2) only frequency and phase are considered. The second filter is applied, when the center point of the movement has already been identified; this yields faster convergence and thus reduces the observation time.

$$
\begin{aligned}
\kappa_{\text {full }}(k T) & =\left[\begin{array}{c}
{[x, y, z]_{\text {center }}} \\
\text { radius } \\
\varphi=\omega k T+\varphi_{0} \\
\omega
\end{array}\right] \\
\kappa_{\text {reduced }}(k T) & =\left[\begin{array}{c}
\varphi=\omega k T+\varphi_{0} \\
\omega
\end{array}\right]
\end{aligned}
$$

The target has to be completely in view by the camera throughout the trajectory estimation. This results 
in a minimum distance of the camera relative to the target and determines the $\mathrm{mm} /$ pixel ratio, i.e. the measurement resolution. In our case at a distance of $d=550 \mathrm{~mm}, 1$ Pixel equals $1.63 \mathrm{~mm}$ in the target plane. This limits the accuracy of the object pose estimation to $\pm 1.63 \mathrm{~mm}$, since sub-pixel processing was not feasible because of the real-time constraint. This measurement error might be reduced by the kalman filter of the trajectory estimator if it would be disturbed by unbiased white noise only. Unfortunately this proved to be not the case, especially because of the non-continuous lighting conditions. The typical overall position error was measured at $2 . .4 \mathrm{~mm}$.

Thus the desired maximum error at the point of insertion of $1 \mathrm{~mm}$ could not be achieved with a single step approach. Therefore a second step was added, which observed the position error just before inserting the connecting rod into the cylinder $(d=150 \mathrm{~mm})$. This time a simple feature-based visual servoing algorithm in the $x-y$-plane was used, which was superimposed on the results of the trajectory estimator. This yielded a maximum position error $<1 \mathrm{~mm}$ relative to the cylinder axis at optimal lighting conditions.

\section{The compliant DLR F/T-Sensor}

The newly developed $\mathrm{f} / \mathrm{t}$-sensor (see figure 6) is based on the already proven SpaceMouse ${ }^{T M}$ technology. The features of this sensor are as follows:

- Combination of a 6-degree-of-freedom optoelectronic measurement unit and a mechanical compliance in one body. This means that forces and torques applied to the sensor result in an elastic deformation of springs inside the sensor which can be $\pm 1.5 \mathrm{~mm}$ in translation and $\pm 3.6^{\circ}$ in rotation. Deviations at the tool-tip can be measured and compensated.

- The complete electronics needed for data evaluation is integrated into the sensor's body, so that the sensor directly delivers forces in $[\mathrm{N}]$ and torques in $[\mathrm{Ncm}]$ at a rate of $4 \mathrm{kHz}$.

- The sensor is able to communicate via CANbus and RS232 or RS485 simultaneously

- The stiffness and resolution of the sensor can be customized by changing number and/or stiffness of the used springs

- Mechanical overload protection in all degrees of freedom

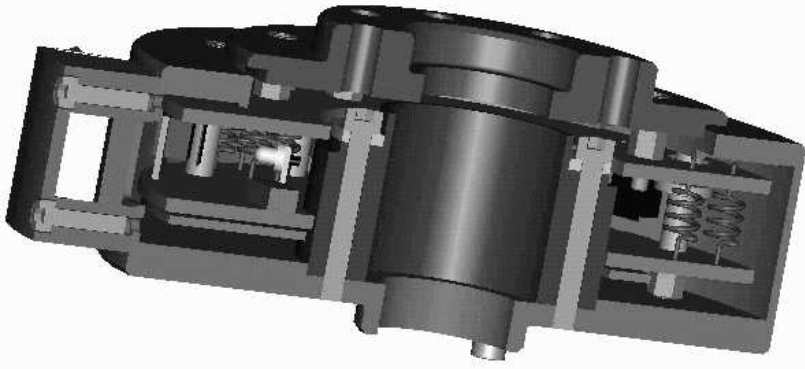

Figure 6: DLR compliant f/t-sensor

The implicit compliant characteristics of the sensor reduce the bandwidth of the occurring contact forces and therefore facilitate the design of efficient force controllers. Position deviations on the sensor due to noncontact forces (e.g. weight of the tool) can be measured and compensated by the robot to keep the tool tip exactly at the desired position even with the mechanical compliance within the kinematic chain.

\section{Force Control}

In order to perform the assembly without damaging the pistons and cylinder contact surfaces a proper control algorithm of contact forces and torques is necessary. In our case, a position-based force control is active at any time the robot end-effector comes in contact with mating parts. It is worth noticing that a position-based force control strategy has been adopted for two main reasons:

- Most industrial robots only provide a position interface

- As proven by many experiments, it is a wellknown concept that if the bandwidth of the inner position loop is much faster than the bandwidth of the outer force control loop, the capability to reject disturbances is larger compared to direct force control [2].

On the other hand, it is clear that whenever the robot has to interact with a rigid surface, its motion is constrained and the only way to control the contact force is to directly control actuator torques [7].

In our application all contact surfaces are rigid. But the robot is equipped with a compliant $\mathrm{f} / \mathrm{t}$-sensor and therefore the end-effector motion is not constrained but its position is directly related to the contact forces through the sensor stiffness. Consequently, it is still 


\begin{tabular}{|l|l|}
\hline Robot time constant & $0.015[\mathrm{~s}]$ \\
\hline Input dead time & $0.020[\mathrm{~s}]$ \\
\hline Sampling time & $0.004[\mathrm{~s}]$ \\
\hline Sensor stiffness & $80[\mathrm{~N} / \mathrm{mm}]$ (x lin.) \\
& $80[\mathrm{~N} / \mathrm{mm}]$ (y lin.) \\
& $80[\mathrm{~N} / \mathrm{mm}]$ (z lin.) \\
& $100[\mathrm{Nm} / \mathrm{rad}]$ (x rot.) \\
& $100[\mathrm{Nm} / \mathrm{rad}]$ (y rot.) \\
& $100[\mathrm{Nm} / \mathrm{rad}]$ (z rot.) \\
\hline \hline Desired Performance & Fast \\
Type of Contact & Stiff \\
\hline
\end{tabular}

Table 1: Technology parameters and desired performance of the robot.

possible and convenient to make use of a positionbased force controller.

\section{Controller Design}

Experiments show that industrial manipulators can be described by simplified models within the range of $10-15 \mathrm{~Hz}$ bandwidth [4]. Such a simplified model can be parameterized by a few but important technology parameters, which can be easily measured or provided by the robot manufacturer (see table 1).

Based on this model a simple but effective automatic procedure can be implemented to design structure and parameters of the controller. Thus the controller design can easily be performed at non-expert level. The automated design has two main targets, namely performance and robustness of the closed loop system. The user specifies a desired performance in terms of response speed and type of contact (see table 1). Once the technology parameters have been provided, the design algorithm computes the controller structure and parameters, guaranteeing high gain and phase margins of the closed loop system. The controller structure chosen by the design routine with the parameters and specifications illustrated in Tab. 1 is a Phase-Lead-Lag compensator. A detailed description of the design procedure with experimental results can be found in $[8]$.

\section{Control Strategy}

To successfully execute the specified task a proper assembly strategy is as important as a robust and efficient force controller. In a first step the assembly strategy involves a classification algorithm which detects the actual state of the assembly process (piston jammed?, which kind of jamming? etc.). The second step is to generate a suitable movement of the robot that helps the system to complete the task.

An assumption for intuitive strategy considerations is to transform all measured forces and torques at the tip of the piston [9]. Therefore all referenced forces are assigned in the end-effector frame.

As soon as the vision system finishes its work, a constant desired force is assigned along the axis of the piston, while a zero set-point is imposed along the other degrees of freedom. In this way, the robot starts moving trying to insert the slightly elliptic piston into the moving cylinder. Depending on the initial positioning accuracy provided by the vision system (typically between $0.5-2 \mathrm{~mm}$ ) two main cases may occur: the piston perfectly fits into the cylinder or it gets stuck on its rim.

Since cylinder and piston axes are surely aligned in the first case, no other forces can build up except the contact friction. Therefore the force set-point is doubled after the piston has been inserted $5 \mathrm{~mm}$ to speed up the insertion process (see fig. 8).

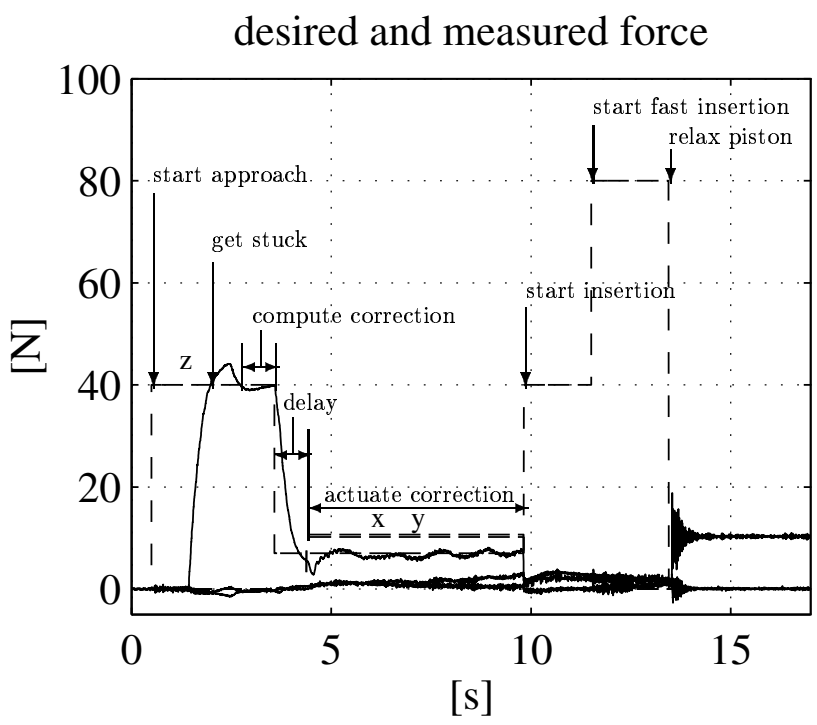

Figure 7: Desired (dashed) and measured (solid) force

In the second case the control strategy must provide a proper action to lead the system to the first case. With reference to fig. 7 , where experimental results in such a case are illustrated, it is easy to recognize the first approach phase when a $40 \mathrm{~N}$ set-point has been assigned and then the time instant when the piston gets stuck on the cylinder rim. When the piston contacts the cylinder rim the resulting contact torques are measured. Considering the given contact geometry it is easy to see that the displacement of the cylinder and 
piston axes is in direction orthogonal to the measured torque (fig. 9). Therefore a correction of the piston's position can easily be achieved by assigning a desired force orthogonal to the measured contact torque so that the piston starts moving to align the cylinder and piston axes.

In order to facilitate the motion of the piston over the engine-block surface, the contact force should be limited to a small value, hence the desired force along $\mathrm{z}$ is set to only $8 \mathrm{~N}$, just to avoid the loss of contact. Correction movements are commanded until the piston axis and cylinder axis are aligned and thus the resulting force in $\mathrm{z}$ direction changes rapidly. To detect this moment, a digital filter with threshold on the output has been implemented to measure the force derivative for enhancing the rapid changes in the measured force.

After a few seconds (depending on the initial error), the piston starts entering into the cylinder, thus the correction has been stopped and the force set-point along the insertion direction has been set back to $40 \mathrm{~N}$. In this way, the system has been led back to the first case, hence it is possible to activate the previous strategy for the fast insertion.

The piston is released after an insertion depth of $20 \mathrm{~mm}$ (see fig. 8). The non-zero steady-state value of the measured force is equal to the piston weight, which was compensated during the whole insertion phase.

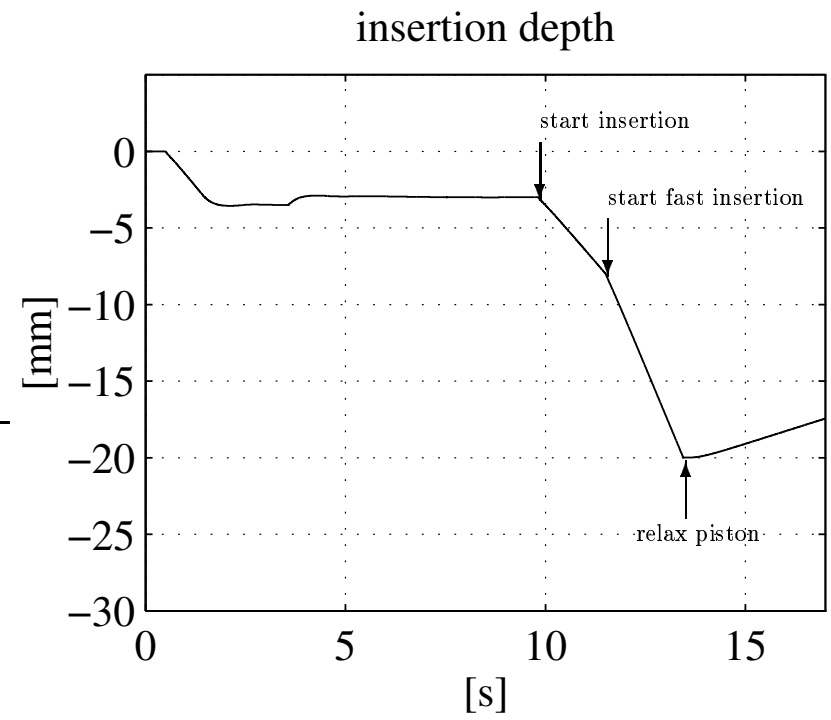

Figure 8: End-effector displacement along z

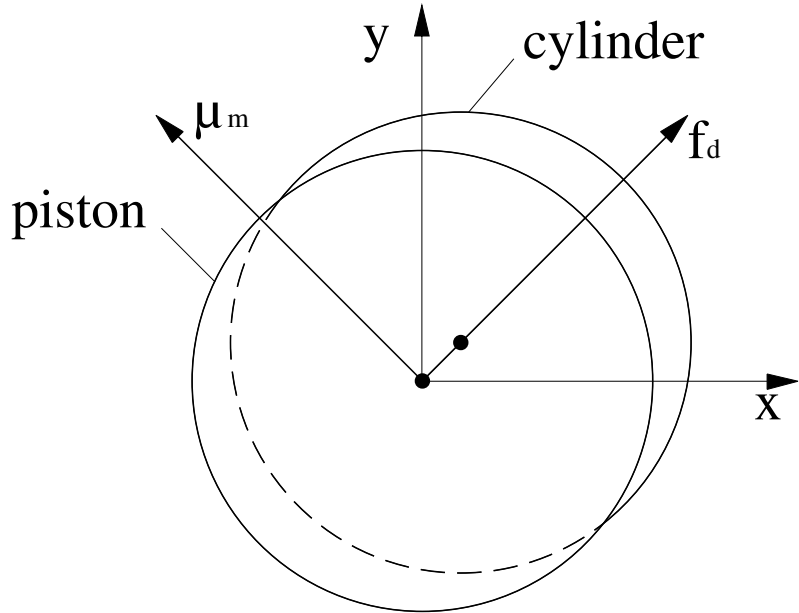

Figure 9: $\mu_{\mathrm{m}}$ : measured torque; $\mathrm{f}_{\mathrm{d}}$ : desired force

\section{Summary and Conclusions}

Our vision-force controlled assembly on moving parts (see fig. 1) was demonstrated at the 1999 Hannover fair, the world's biggest industrial fair, and soon turned out to be one of the most attractive highlights among all automation related exhibits. A video of this demonstration was accepted for presentation at the ICRA 2000 conference [6]. We proved that complex automation problems can be solved with sensorcontrolled robots. Choosing robust methods for image processing and force control combined with heuristic strategies yields a good performance. Future work will be:

- development of compliant sensors with greater measurement range (up to $250 \mathrm{~N}$ )

- higher robustness with regard to lighting conditions

- higher sampling rates and interpolation cycles yielding shorter handling time

- fully integration of the image processor into the robot controller

- allow mounting at any angle

- integration of a laser scanner

\section{Acknowledgments}

This development was supported by the German Federal Ministry for Technology in the framework of the project LISSY (Production 2000). 


\section{References}

[1] K. Arbter, J. Langwald, G. Hirzinger, G.-Q. Wei and P. Wunsch, "Proven Techniques for Robust Visual Servo Control," in Robust Vision for Vision Based Control of Motion, IEEE Press, (to appear).

[2] J. De Schutter, H. Bruyninckx, H. Zhu, M. W. Spong, "Force Control: A Bird's Eye View," in Control Problems in Robotics and Automation, Siciliano B. and Valavanis $K$. R.(Eds.), Springer Verlag, pp. 1-17, 1998.

[3] E. D. Dickmanns and V. Graefe, "Dynamic Monocular Machine Vision," Machine Vision and Applications, vol. 1,pp. 223-240,1988.

[4] G. Hirzinger, "Robot-Teaching via Force-TorqueSensors," Proc. of the Sixth European Meeting on Cybernetics and Systems Research, Vienna, A, pp. 955-960, 1982.

[5] P. V. C. Hough, "A Method and Means for Recognizing Complex Patterns," U.S. Patent 3,069,654, 1962 .

[6] S. Jörg, J. Langwald, J. Stelter, G. Hirzinger and C. Natale, "Flexible Robot-Assembly using a Multi-Sensory Approach," Video Proc. IEEE International Conf. on Robotics and Automation, San Francisco, CA, 2000.

[7] H. Mc Clamrock, J. Whang, "Feedback Stabilization and Tracking of Constrained Robots," IEEE Trans. on Automatic Control, vol. 33, pp. 419$426,1988$.

[8] C. Natale, R. Koeppe, G. Hirzinger, "An Automatic Procedure for Force Controller Design," 1999 IEEE/ASME Int. Conference on Advanced Intelligent Mechatronics, Atlanta, GA, (to appear).

[9] D. E. Whitney, "Quasi-Static Assembly of Compliantly Supported Rigid Parts," ASME J. of Dynamic Systems, Measurement, and Control, vol. 104, pp. 65-77, 1982.

[10] P. Wunsch, G. Koegel, K. Arbter and G. Hirzinger, "Kalibrierung eines nichtlinearen, binokularen Hand-Auge Systems," German Aerospace Center - DLR, Technical Report No. 515-96-27.
[11] P. Wunsch and G. Hirzinger, "Real-Time Visual Tracking of 3-D Objects with Dynamic Handling of Occlusion," 1997 Proc. IEEE International Conf. on Robotics and Automation, Albuquerque, NM, 1997.

[12] P. Wunsch, "Modellbasierte 3-D Objektlageschätzung für visuell geregelte Greifvorgänge in der Robotik," Dissertation, University of Technology, Munich 1997, Shaker-Verlag, ISBN3-8265-3267-8, 1998. 